\title{
Surface Modification of Polymers for Tissue Engineering Applications: Arginine Acts as a Sticky Protein Equivalent for Viable Cell Accommodation
}

\author{
Poulomi Sengupta and Bhagavatula L. V. Prasad*(i) \\ Physical Chemistry Division, CSIR National Chemical Laboratory Dr. Homi Bhabha Road, Pashan, Pune 411008, India
}

Supporting Information

\begin{abstract}
Hydrophobic polymers, for their favorable mechanical properties, are a popular choice as permanent bioimplants. These materials remain absolutely bioinert for years, but throw up challenges when it comes to fast integration with healthy tissue. Addressing this, herein, we present a surfacemodification technique of converting the hydrophobic surface of a polymeric film into a hydrophilic one using a layer-by-layer assembly process involving gold nanoparticles and small molecules like amino acids. These films showed much improved animal cell (murine fibroblast) adherence properties compared to commercially available tissue culture plates. Moreover, arginine-modified films exhibited a nearly equivalent cell viability compared to the films modified with the natural extracellular matrix component fibronectin. The surface hydrophilicity and roughness of our novel film were characterized by contact angle measurement and atomic force microscopy. Cell counting, fluorescence microscopy, cell viability, and collagen estimation assay were employed to demonstrate that our film favored a much improved cell adherence, and accommodation in comparison to the commercially available tissue culture plates.
\end{abstract}

\section{INTRODUCTION}

Synthetic polymeric scaffolds are being extensively used as tissue replacements inside the human body. ${ }^{1}$ Load-bearing organs such as the hip joint and the knee joint are regularly replaced with either polymer or polymer-metal composites. Compared to polymer-metal combinations, pure polymeric materials have several advantages, as they can be molded into different shape/size/porosity. For example, polymers like polyethylene have a very long history of being "the material of choice" as implant applications for their right strength and optimal hardness. ${ }^{2,3}$ Conversely, being hydrophobic, these polymers are nonresponsive toward tissue integration, which may lead to dislocation and fibrosis, followed by multiple corrective surgeries. ${ }^{4-6}$ One way to overcome this impediment is surface modification, where the bulk properties (mechanical strength, elasticity) of a material remain unchanged, whereas small modifications in the surface impart necessary characteristics, allowing a faster integration of the implant with the tissue. In this context, surface modification of the polymers with proteins (fibronectin, vitronectin, laminin) present in the extracellular matrix (ECM) is a regularly followed strategy. ${ }^{7,8}$ These proteins include a specific tripeptide sequence RGD (arginine-glycine-aspartic acid) that is very specifically recognized by the cell surface receptor protein integrin. It has been widely reported in the literature that fibronectin-coated polymeric scaffolds portray enhanced cellular adherence, leading to a drastically improved cellular proliferation. ${ }^{9,10}$ To avail the benefits of both polymer (as material) and fibronectin/vitronectin (as surface modifying agents), an even coating of ECM protein on polymer will be ideal. But, the polymer of choice being hydrophobic, uniform surface modification with a protein is impossible, exclusive of multistep chemical interventions. In this context, the layer-by-layer ${ }^{11-14}$ assembly is emerging as a relatively simpler technique that can produce a ubiquitously uniform surface modification. Previous reports from our group have already established that plasma treatment of hydrophobic polymeric films followed by layer-bylayer assembly of gold nanoparticle and lysine can "lock" the hydrophilic characteristics of the surface and increase the cell friendliness of the surface and promote cell growth. ${ }^{15,16}$ This method is unquestionably superior to sole $\mathrm{NH}_{3}$ plasma treatment due to the prolonged retention of hydrophilicity. Moreover, such a modified surface works as an excellent support for cell attachment and proliferation. At room temperature, the films that were modified following the above strategy were found to retain the hydrophilic characteristics for more than 1 year. In contrast, with only plasma treated films, the hydrophobic character got regenerated within a week. ${ }^{17}$

In this report, with the aim of creating a cell-friendly surface out of the readily available materials by a simple and convenient

Received: February 5, 2018

Accepted: April 9, 2018

Published: April 16, 2018 


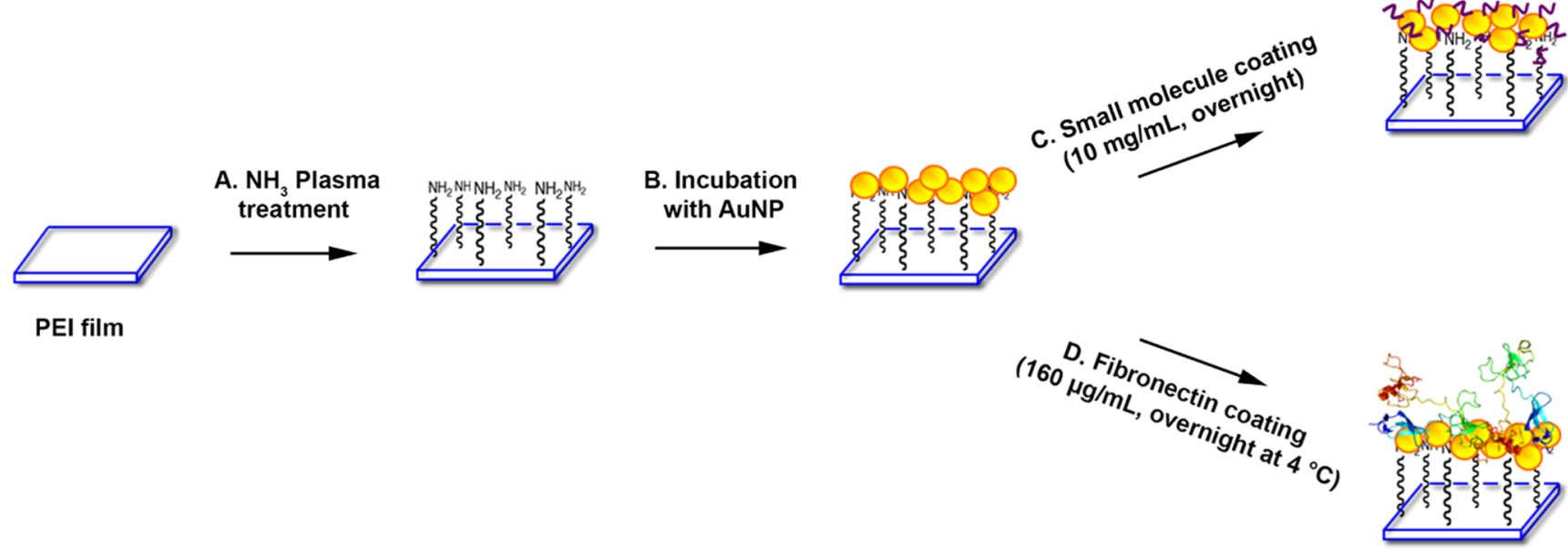

Figure 1. Schematic representation of surface modification for polyetherimide (PEI) films. (A) Plasma treatment on PEI films using 3:2 hydrogen and nitrogen mixture. (B) Citrate-stabilized gold nanoparticles incubation with plasma-treated films. (C) Dip coating of films inside the argininewater solution overnight. (D) Gold-coated films were incubated with a dilute solution of fibronectin.

technique, we have surface-modified plasma-treated polyetherimide (PEI) films via layer-by-layer assembly of gold nanoparticles and arginine. This has been achieved by simple overnight incubation of the plasma-treated films in a gold sol followed by dip coating of arginine (Figure 1). We then evaluated the applicability of these films for murine fibroblast cell L929 adherence and proliferation. As a positive control, we have taken fibronectin-coated (on gold-coated films) films (Figure 1) and compared several properties like cellular adhesion and proliferation, the quantification of live cells, the expression of F-actin, and the quantification of collagen. To our surprise, we have found nearly comparable results in both the treatments, which led to the conclusion that the small molecule arginine can be considered to be a sticky protein equivalent when it comes to viable cell population on a surface-modified polymeric film. To rationalize our observation, we also have shown that L929 cells grown on both arginine- and fibronectincoated films produce an equivalent amount of collagen on prolonged culture, indicating that the cellular accommodation mechanism with both these molecules is probably the same.

\section{RESULTS}

Characterization of Films. Pristine and surface-modified films were characterized using UV-vis, IR, atomic force microscopy (AFM), and dynamic mechanical analyzer (DMA). The as-prepared Au NP sol was prepared by the Turkevich method ${ }^{18}$ and displayed a peak in the UV-vis spectrum at $530 \mathrm{~nm}$ (Figure S1A). The transmission electron microscopy (TEM) analysis indicated that the particles are around $20 \mathrm{~nm}$ (Figure S1B). On the other hand, the films coated with gold nanoparticles displayed a peak at $580 \mathrm{~nm}$ (Figure S2A,B) in the UV-vis spectrum acquired. The Supporting Information Figure S3 provides the contact-mode AFM images, which highlight the transformation in the surface structure and the roughness of the film consequent to surface modification. In the case of pristine PEI film, the surface roughness parameter was estimated to be $R_{\mathrm{a}}=9.85 \mathrm{~nm}$ (Figure $S 3 A)$. For gold-coated films, the roughness value increased to $\sim 50 \mathrm{~nm}$ (Figure S3B). Interestingly, when the same films were surface modified with arginine (Figure S3C), the surface roughness parameter increased drastically $\left(R_{\mathrm{a}}=107 \mathrm{~nm}\right)$. On the other hand, fibronectin coating on the gold-coated PEI surface did not change the surface roughness parameter (Figure S3D) very much, although a little elevation in its value $\left(R_{\mathrm{a}}=\right.$ $66.1 \mathrm{~nm})$, when compared to only gold-coated films was observed.

In Figure 2, the Fourier transform infrared (FT-IR) data (attenuated total reflectance (ATR) mode) for pristine PEI

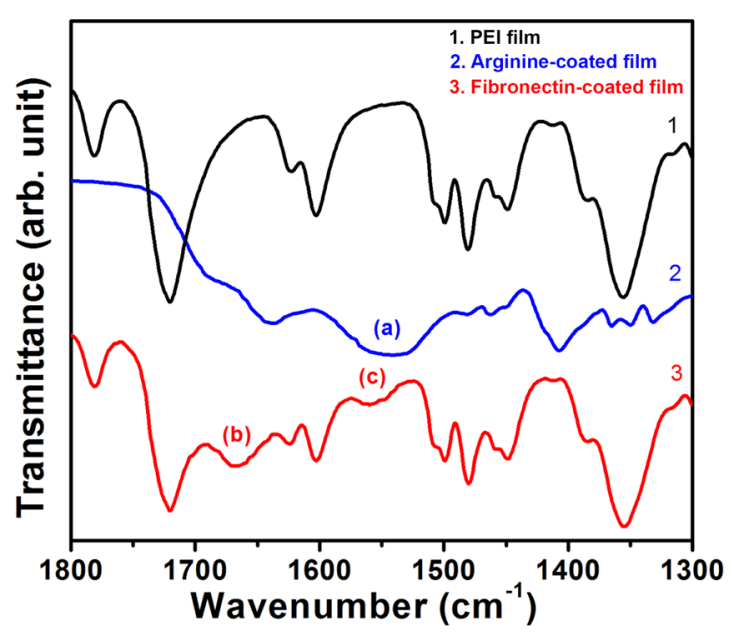

Figure 2. FT-IR spectrum taken in the ATR mode for the characterization of signature peaks from (1) PEI film (2) argininecoated PEI film, and (3) fibronectin-coated PEI film. (a) Broad peak at $1550-1600 \mathrm{~cm}^{-1}$ corresponds to protonated, hydrated guanidium groups. (b) Amide I (from fibronectin) $1670 \mathrm{~cm}^{-1}$ and (c) amide II $1560 \mathrm{~cm}^{-1}$.

film, arginine-coated gold-PEI film, and fibronectin-coated gold-PEI film in the $1300-1900 \mathrm{~cm}^{-1}$ range have been consolidated. Curve 1 (black line) shows the FT-IR spectrum of the pristine PEI film. Curve 2 (in blue) corresponds to the arginine-coated gold-PEI film, where the stretching motion of the CO group at $1630 \mathrm{~cm}^{-1}$ could be seen. The band at 1520 $\mathrm{cm}^{-1}$ was assigned to the bending vibration of $-\mathrm{OH}$ (indicated as peak a). Further, the sharp peak at $1410 \mathrm{~cm}^{-1}$ corresponds to the symmetric in-plane bending, and the asymmetric bending of $\mathrm{CH}_{3}$ groups could be seen around $1450 \mathrm{~cm}^{-1}$. The fibronectincoated films when probed with FT-IR (curve 3, red line) showed very specific amide I peak at $1670 \mathrm{~cm}^{-1}$ (b) and amide 
II peak at $1560 \mathrm{~cm}^{-1}$ (c). The full-range IR spectrum is provided in the Supporting Information Figure S5.

The "contact angle measurement" is a strong tool for the determination of hydrophilicity and, accordingly, we measured the contact angles after different surface-modification procedures, which are presented in Figure 3. As can be noticed, the

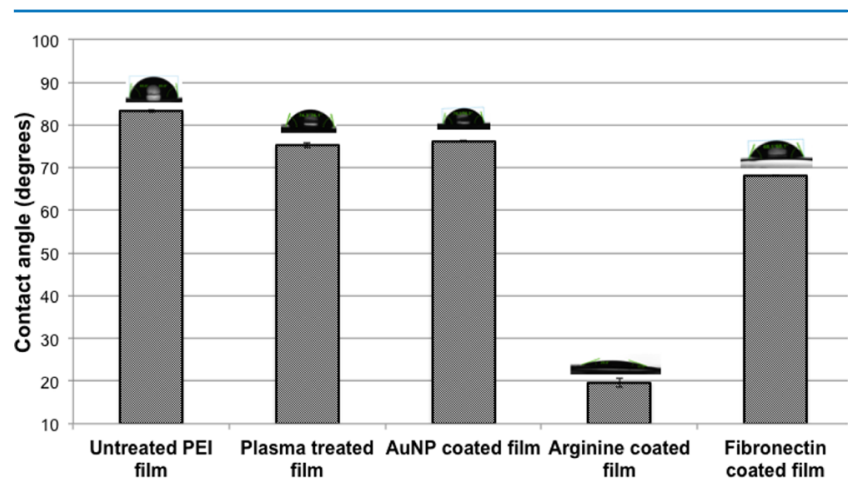

Figure 3. Contact angle determination using $10 \mu \mathrm{L}$ water droplet on the treated film. The data represented $\pm \mathrm{SD}$ of $n=3$.

pristine PEI films exhibited a contact angle value of $83^{\circ}$ with 10 $\mu \mathrm{L}$ of water at the air-water interface. Upon $\mathrm{NH}_{3}$ plasma treatment and citrate-stabilized gold nanoparticle coating, the contact angle decreased to $\sim 75^{\circ}$. The arginine-coated films were extremely hydrophilic, having a contact angle in the low range around $\sim 19^{\circ}$. The fibronectin-coated film, on the other hand, was more hydrophobic, having a contact angle value of $\sim 68^{\circ}$.

A set of water absorption studies were carried out, which have revealed that the arginine-coated film held significantly more amount of water on the surface in comparison to the fibronectin-coated or the bare film. The results are presented in Figure S4. We also checked the tensile strength of the films after different surface modifications using DMA (Figure S6). It may be noticed that in all the cases (the bare PEI, argininecoated, and fibronectin-coated films), there was no significant change in the Young's modulus value (value lies in the range of 1000-2000 MPa). Till the application of $30 \mathrm{MPa}$ pulling force, no breaking of the film was experienced.

Cell Adhesion. Cellular Adhesion Study. From the cell count experiment using optical microscopy (Figure 4), it can be clearly seen that with the increase in time, more and more murine fibroblast cells get adhered to the arginine-coated film.

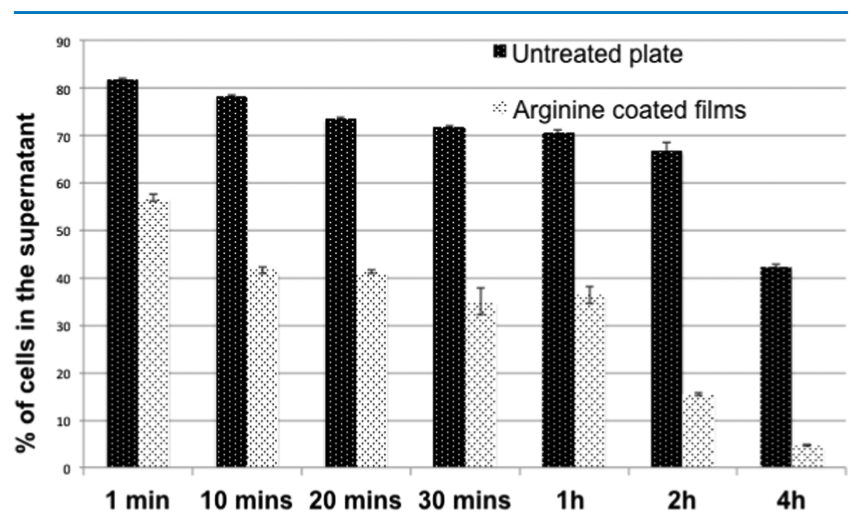

Figure 4. Cellular adhesion experiment by cell counting where notadhered cells were estimated using Trypan blue. Data represented as a $\pm \mathrm{SD}$ of $n=3$.
The number of cells present in the supernatant decreased with time, indicating that more than $40 \%$ of the cells adhered to the arginine-coated films within $1 \mathrm{~min}$, whereas in control nontreated plate, less than $20 \%$ cells adhered. Similarly, within $1 \mathrm{~h}$, almost $60 \%$ cells adhered to the arginine-coated films, whereas the extent remained around $30 \%$ for the bare plate. At $2 \mathrm{~h}$, the change was drastic, when almost $85 \%$ cells adhered to the arginine-coated films and only around $35 \%$ cells adhered to the control plate. In summary, we observed that almost all the cells were found adhered to the arginine-coated films at $\leq 2 \mathrm{~h}$.

Cell Viability and Cytotoxicity. Cell Viability Study by Mitochondrial Activity. In this experiment (Figure 5), viable

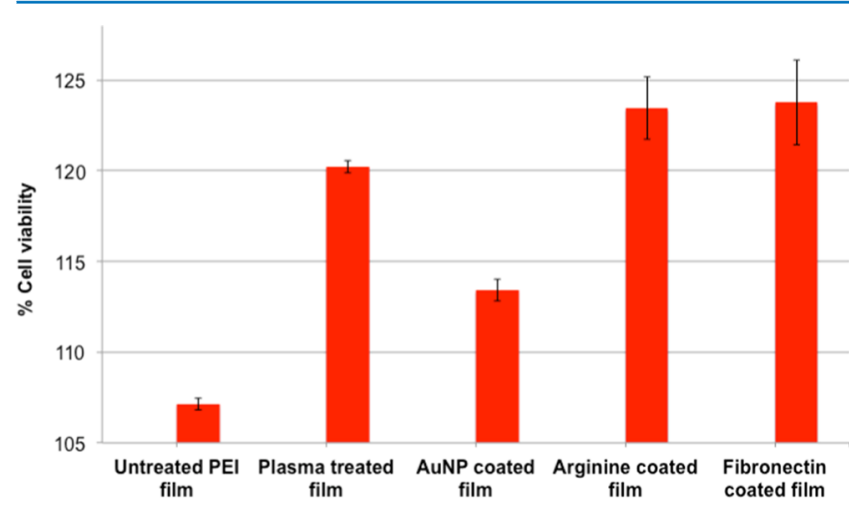

Figure 5. Cell viability by mitochondrial activity using resazurin indicator at $48 \mathrm{~h}$. Data represented as $\pm \mathrm{SD}$ of $n=3$.

cells were estimated by mitochondrial activity, as indicated by the reduction of resazurin to resofurin (emission at $590 \mathrm{~nm}$ ). The data presented here were normalized with respect to the untreated plate value. In accordance with the cell-counting experiment, it was observed that both fibronectin- and argininecoated films allowed cell proliferation from 122 to $125 \%$ at the end of 2 days. However, for all the other controls, the value was less. The plasma-treated films also had almost $120 \%$ viable cells. The normalized data show that even with pristine PEI, the cell viability was above $100 \%$.

Cell Viability by Live-Dead Imaging. Propidium iodide (PI) and acridine orange dyes were used for the analysis of dead and alive cells present on the film surface, respectively. Here also, the arginine-coated films showed an increased number of healthy cells population, but there were few dead cells (Figure 6) as well. On the other hand, the fibronectincoated films showed excellently adhered cells and no dead cells were present according to the PI staining. The emission intensity from two fields green (representing live cells stained by acridine orange) and red (representing dead cells stained by propidium iodide), as shown in Figure S7A, also supports this conclusion.

Cellular Proliferation Analysis. Cellular Proliferation Study by Cell Counting. In this experiment, at the 1 day time point, arginine- and fibronectin-dipped gold-coated PEI films were found to produce almost equal number of live cells (Figure 7, blue columns). At 2 days time point as well, the arginine- and fibronectin-coated films behaved almost similarly (Figure 7, red columns), whereas gold-coated and pristine films showed a lower cell population. Similarly, fresh plasma-treated films also indicated a reasonably high number of cellular population.

Cellular Population by Fluorescence Microscopy. Using this method, we visualized the cellular density of the adhered 


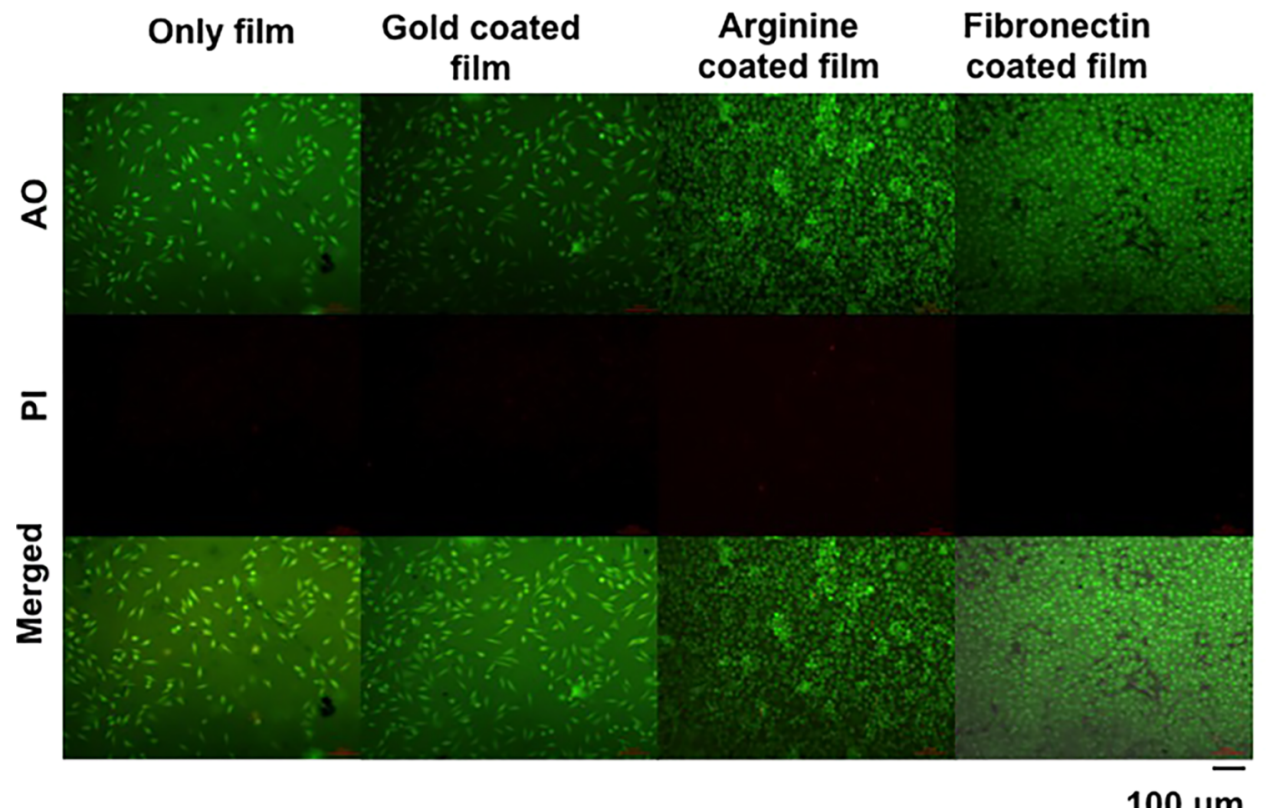

$100 \mu \mathrm{m}$

Figure 6. Live-dead assay by live cells imaging. Live and dead cell stains are acridine orange (AO) and propidium iodide (PI), respectively. The scale bar is $100 \mu \mathrm{m}$.

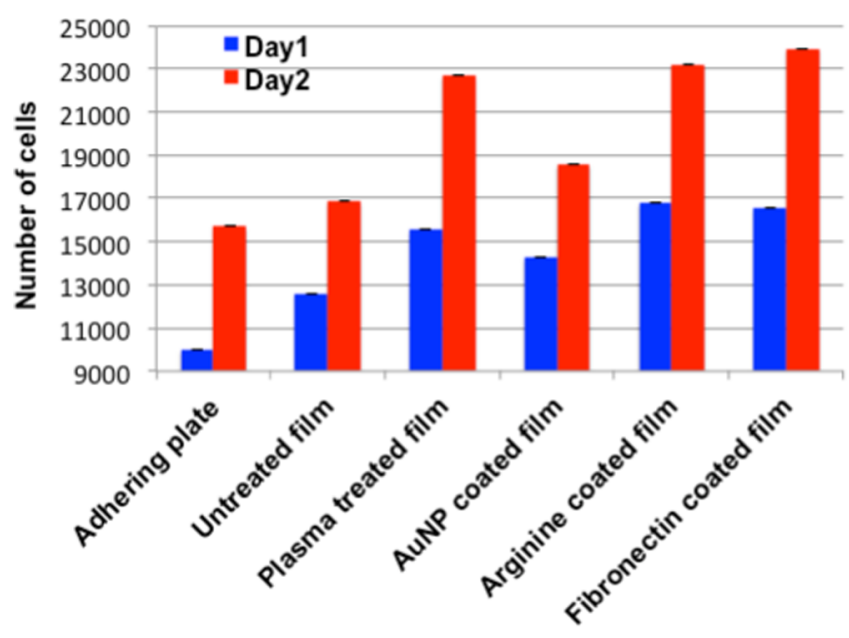

Figure 7. Cell proliferation study by cell counting using Trypan blue exclusion test (data represented as the mean \pm SD of $n=3$ ).

cells on each surface-modified and control film. For pristine PEI films (Figure 8), the cellular density was low. For the goldcoated surfaces, the cellular density was more than that of the pristine films but definitely less than that of the plasma-treated films. For both arginine- and fibronectin-coated films, the cellular density was rather high and uniform. However, the fibronectin-coated films showed a more sticky behavior for the fibroblast cells. Again, the measurement of fluorescence intensity from green and blue channels represented a reasonably high cellular density and their healthiness with both ariginine and fibronectin surface modifications (Figure S7B).

Cellular Morphology by Actin Cityscape's Staining. According to Figure 9, the arginine-coated films indicated an elongated disposition of actin filaments, which confirmed the healthy and happy condition of the cells. In fibronectin-coated films as well, the cells appeared elongated and stretched. A closer look at the cellular adhesion (Figure S8) showed that
L929 cells present on arginine-coated films are elongated. At the same time, they are a little folded at sides, which was absent in the case of the fibronectin-coated films.

Production of Collagen. In this experiment, we measured the amount of collagen expressed both from cellular layer and spent media. The collagen estimation kit works on the principle of oxidation of both proline and hydroxyproline present in the ECM, which is directly proportional to the amount of collagen produced by the healthy cells. From the cell pellet (Figure 10A), the estimated collagen from both arginine- and fibronectin-coated films were comparable in amount, whereas in control samples, the amount was significantly less. The data were plotted after normalizing with "only film" values, which indicated that collagen produced in the arginine- and fibronectin-coated films was 3 times higher than that present on the pristine film (Figure 10B). Even in the spent medium, a similar trend is followed, which is in accordance with the cell viability and cell count data.

\section{DISCUSSION}

The gold nanoparticle surface chemistry opens up a big horizon for the functionalization of the polymeric solid scaffolds. Our strategy to surface modify the polymeric films (which could be easily extended to porous scaffolds) was initiated with the plasma treatment of a hydrophobic polymeric film. For the plasma treatment, we used a mixture of $\mathrm{H}_{2} / \mathrm{N}_{2}$ gases, which was expected to generate ammonia plasma ${ }^{19,20}$ in situ. This reactive ammonia plasma interacts with the surface of the polymers generating hydrophilic functional groups ${ }^{21}$ (mostly $-\mathrm{NH}_{2}$ groups). These films were then dipped in a gold nanoparticle hydrosol. These gold nanoparticles were in turn prepared by the well-established citrate-reduction method. ${ }^{18}$ The asprepared gold nanoparticle sol has a characteristic wine-red color displaying an absorbance peak at $\sim 520 \mathrm{~nm}$ in the UV-vis spectrum. The absorbance is attributed to the localized surface plasmon resonance. Subsequently, the simple dipping of the plasma-treated PEI films in gold nanoparticle hydrosol leads to a quick and firm attachment of the nanoparticles to the plasma- 


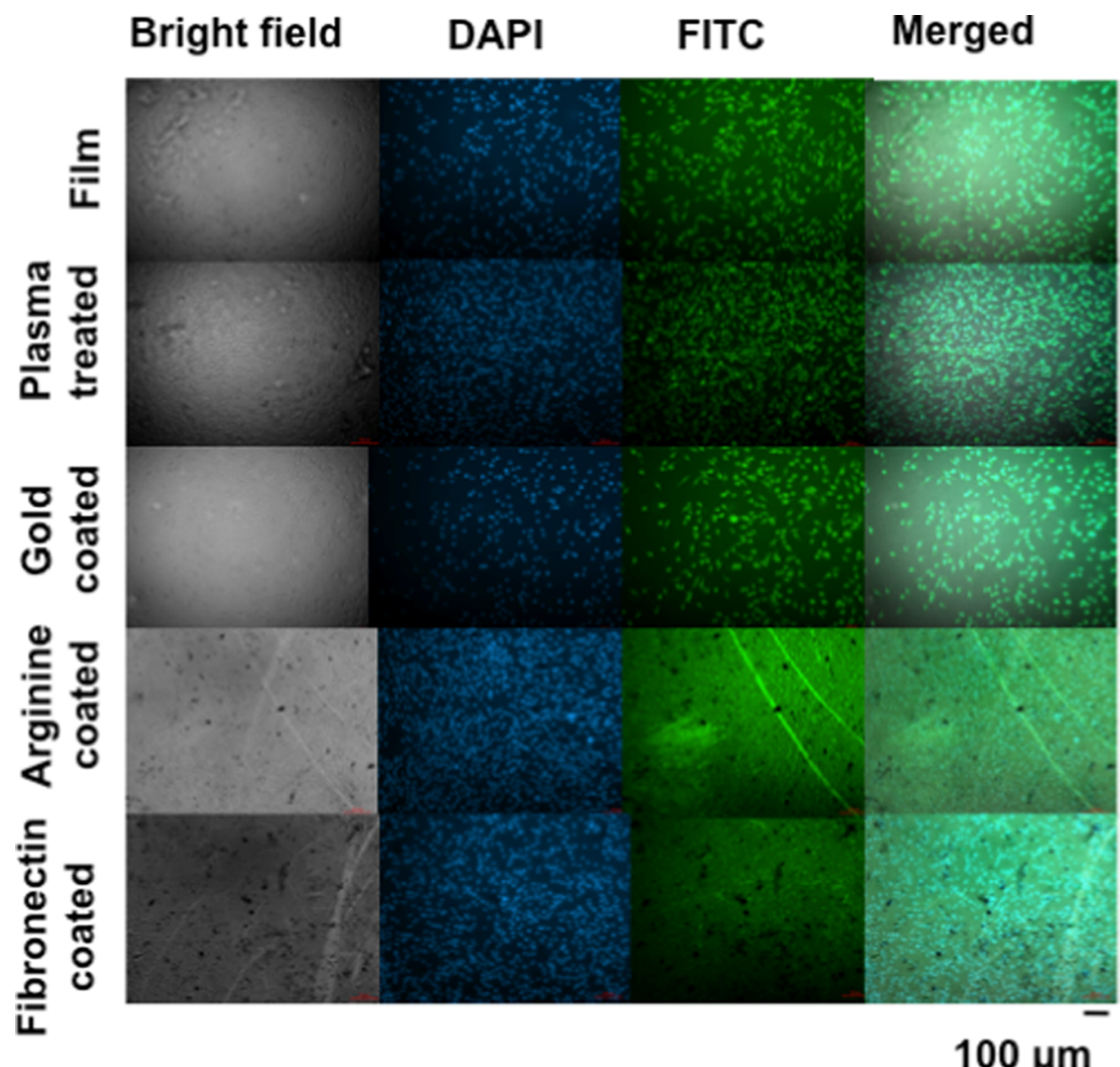

Figure 8. Nucleus and cytoplasm staining of L929 fibroblast cells using fluorescence microscope. Scale bar is $100 \mu \mathrm{m}$.

treated film surface due to the electrostatic attraction between the positive and negative charges of amine and citrate groups. This contention was supported by the UV-vis spectrum of the gold nanoparticle adhered film, which shows a peak at $580 \mathrm{~nm}$ (Figure S2A), which is significantly different from the peak observed for the as-prepared gold sol. This shift in surface plasmon resonance is expected and ascribed to the aggregated nature of the gold nanoparticles on the film. To check how strongly these Au NPs adhere to the PEI film, we immersed the gold-decorated PEI films in water at $90{ }^{\circ} \mathrm{C}$ under stirring conditions. We did not notice leaching of any gold into this water, as confirmed by the absence of the surface plasmon peak of Au NPs in the supernatant. Thinking that, in case any trace amount of Au NPs leached out by hot water treatment, they should dissolve and convert to $\mathrm{Au}^{3+}$ ions in aqua regia, we evaporated the water and added aqua regia to it. We did not find any signature of $\mathrm{Au}^{3+}$ ions from this aqua regia solution. This confirms that no Au NPs got leached into the hot water (these data are not included for brevity).

Our previous results suggest that the primary amine groups of the amino acids electrostatically interact with the citrate groups on the gold nanoparticles. ${ }^{17}$ The interaction is stronger if the amino acids have more than one free amine group, which can have a constructive interaction with the citrate capping present on the gold nanoparticle surface. Although our previous results suggested lysine as a good surface-coating agent, and provided enhanced cell adhesion and proliferation, ${ }^{17}$ as a part of this work several other amino acids were also tried (data not shown). It was concluded that positively charged amino acid arginine adsorbs very strongly to gold nanoparticles, resulting in a super hydrophilic yet rough surface, which is expected to increase the cellular adhesion properties, as confirmed from the contact angle measurements described below.

The $83^{\circ}$ water contact angle on the pure polymer film clearly established that it is considerably hydrophobic. Upon $\mathrm{N}_{2}+\mathrm{H}_{2}$ plasma treatment, the contact angle decreased to $75^{\circ}$, indicating a lowering in the hydrophobic character of the film. The attachment of the citrate-stabilized gold nanoparticle coating on the plasma-treated PEI films changed the contact angle only slightly $\left(76^{\circ}\right)$ (Figure 3 ). The overnight incubation of these films in arginine solution led to a drastic lowering of the contact angle value $\left(19^{\circ}\right)$. This is attributed to the guanidium group present in the side chain of arginine, which is extremely hydrophilic. Once guanidium or any amine group anchors on the gold nanoparticle surface, the other amine or carboxylic acid groups present in arginine will be exposed outward and preferentially interact with water thus explaining the enhanced hydrophilicity. On the other hand, the fibronectin-coated films did not show a great change in the contact angle (changed from $76^{\circ}$ in AuNP-coated film to $68^{\circ}$ ) value. This could be rationalized by considering the composition of fibronectin, which is a combination of both hydrophilic and hydrophobic amino acids. From water absorption studies, it has been confirmed that the arginine-coated films possess a high water retention capability as shown in Figure S4. The bare PEI film, having no surface functionalization, is unable to retain water on 


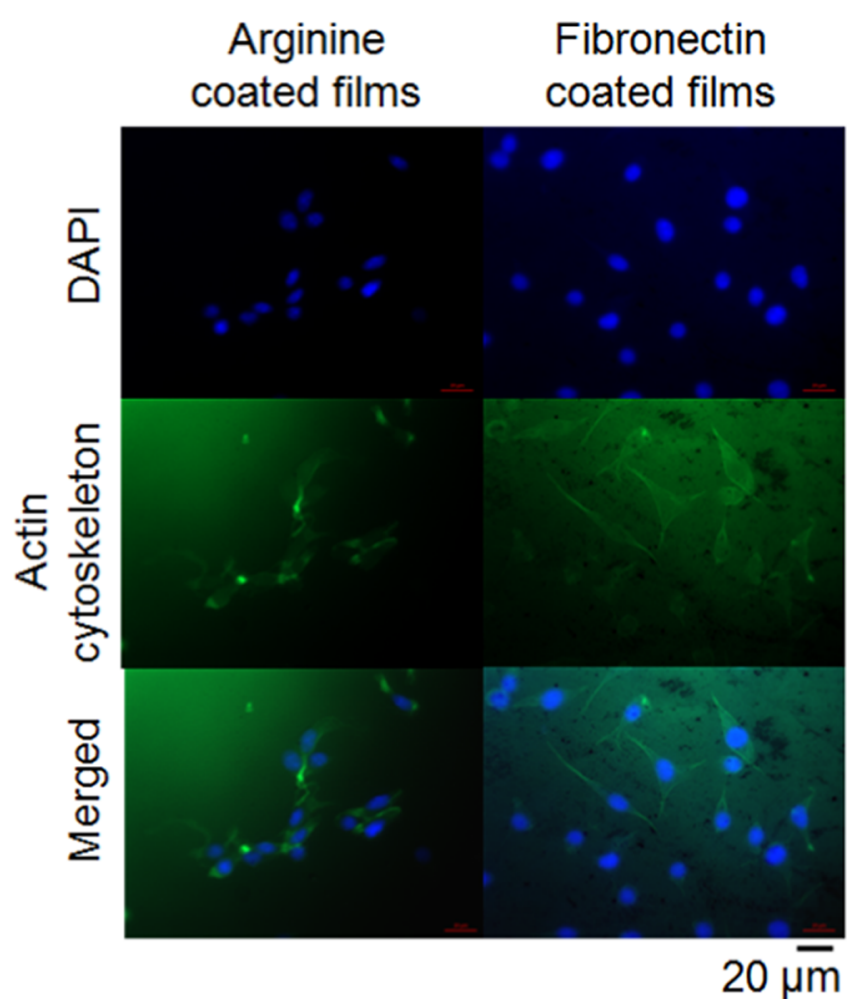

Figure 9. Actin filament staining of L929 cells with Alexafluor 488 phalloidin and 4',6-diamidino-2-phenylindole (DAPI) for nucleus counterstaining. Scale bar $20 \mu \mathrm{m}$.
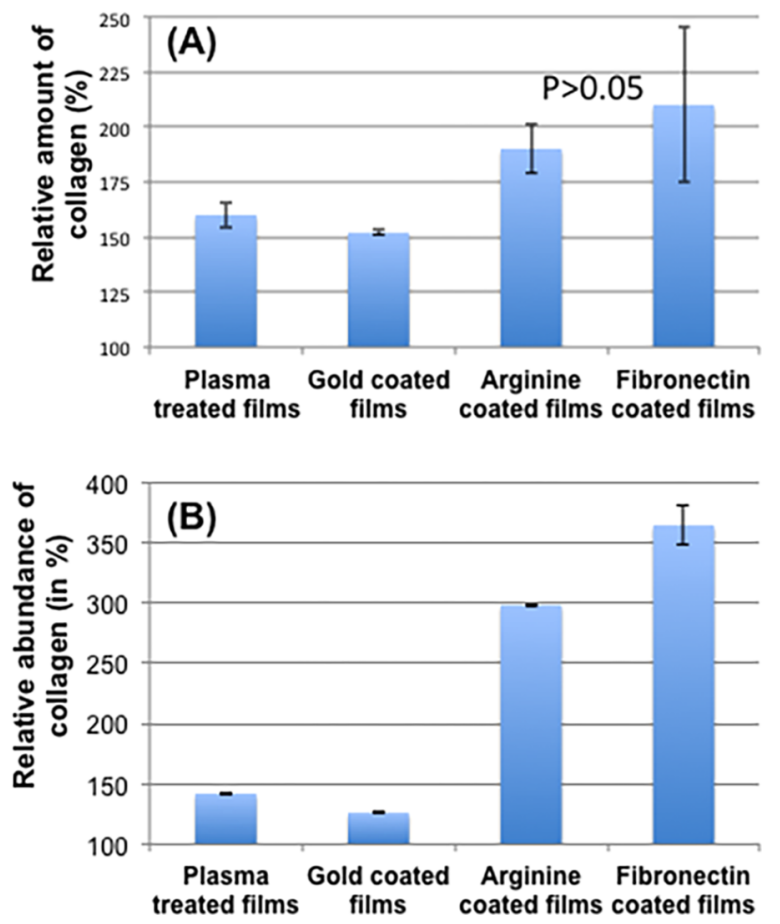

Figure 10. Expression of collagen (A) on the surface of different films (B) in different well's spent media.

the surface. The fibronectin-coated films showed moderate water absorption properties.

The trends in contact angle changes and water absorption data are reflected in the surface roughness characteristics determined from the AFM measurements as well (Figure S3).
The pristine polymer film displayed a roughness value of $R_{\mathrm{a}}=$ $9.85 \mathrm{~nm}$. With arginine, the parameter increased significantly to $107 \mathrm{~nm}$, which may be attributed to the restructuring of the nanoparticles on the polymeric film surface in the presence of arginine (Figure S3C). The change in the nature of nanoparticle adherence on the polymeric surface is also corroborated by the change in the SPR position $(580 \mathrm{~nm}$; Figure $S 2 A, B)$. In the case of the fibronectin-coated films, the surface roughness also increased (Figure S3D), but it is not as high as that in the case of the arginine-coated surface. This may be attributed to a higher molecular entanglement present in protein in comparison to small molecules. The UV-vis results also indicated the retention of the peak at $580 \mathrm{~nm}$ after incubation with arginine and protein, confirming the presence of gold nanoparticles on the polymer surface.

In spite of all these surface modifications, there has not been any significant change in the Young's modulus of the bulk polymeric film, as indicated by Figure S6. The strain in samples (bare PEI film, arginine-, and fibronectin-coated films) steadily increased with increase in stress in the mega pascal area, which signifies that the high mechanical robustness of the polymeric film did not get altered due to surface modification.

Whereas the changes in UV-vis spectra, contact angle, water absorption, and surface roughness values do provide an indication of the changes in the surface characteristics of the films due to the sequential layer-by-layer assembly of gold nanoparticles and the coating of arginine or fibronectin onto them, characterization by IR provides conclusive evidence of the presence of arginine and fibronectin on the films. Solid-state FT-IR (ATR mode) is a robust technique for determining the signatures in surface-modified films. It has to be kept in mind that PEI itself is a chemically complex molecule that has several signature peaks representing either aromatic $\mathrm{C}-\mathrm{H}$ units, or phthalimide groups, etc. The FT-IR spectrum of PEI alone displayed a sharp peak at $1720 \mathrm{~cm}^{-1}$ corresponding to the lactum carbonyl group present in it (curve 1 in Figure 2). Therefore, in all the surface-modified films, a baseline with only PEI was performed. Accordingly, from the FT-IR analysis, it can be very clearly seen that in the arginine-coated films, a broad peak of protonated, hydrated guanidium group appears in the range $1500-1600 \mathrm{~cm}^{-1}$ [curve 2(a), Figure 2]. Also, in the fibronectin-coated films, a strong $\gamma_{\mathrm{N}-\mathrm{H}}$ [amide I and amide II peaks in Figure 2 curve 3(b,c), respectively] was observed, confirming the protein attachment to the AuNP-coated films.

After establishing that the layer-by-layer assembly of gold nanoparticles and fibronectin or arginine can be achieved by simply dipping the PEI films in the respective solutions, we proceeded to evaluate the cellular adhesion capability of the surface-modified (by arginine) films. For this, two different experiments were conducted. In the first experiment, the cellattaching properties of arginine-coated films were evaluated in comparison to nontreated polystyrene plates. The surfacemodified films showed up to $85 \%$ cellular adhesion $\leq 2 \mathrm{~h}$. We attribute this to the hydrophilic character of the arginine-coated films (evident from contact angle, water absorption measurements), roughness (evident from AFM measurements), and the presence of positive charges (from the arginine side chain). The ultimate goal of these surface-modified films is to accommodate healthy cells. So, after establishing that the arginine-coated films support a strong cell adherence, we wanted to check their cell proliferation capability over time. For this purpose, we chose to compare the cell viability properties with a strong positive control: the fibronectin-coated films. 
The amount of mitochondrial activity in viable cells was determined by the reduction of resazurin, which is a standard chemical for this purpose. It may be noticed that after $48 \mathrm{~h}$ incubation, an equivalent amount of viable cells were found in both arginine- and fibronectin-coated films (Figure 5). Plasmatreated films also showed a higher number of viable cells. This can result from the transient surface modification of the polymer surface (due to plasma treatment). As expected, untreated and gold-coated surfaces showed less number of cells. In the case of untreated films, this could be due to their hydrophobic character, whereas in the case of gold nanoparticle-coated films, the negative charge of the citrate molecules present on their surface may be implicated for the cell unfriendliness. We wish to emphasize here that all the data from different films showed a considerably higher cellular proliferation in comparison to nontreated tissue culture plate, which supports the fact that the PEI films per se are not at all cytotoxic.

One point worth noticing in our results is that, in live-dead imaging assay (Figure 6), arginine-coated films showed a few dead cells along with numerous healthy cells. This may arise due to the dearth of space and nutrients, which may impede the growth of cells and pushed the cell cycle toward apoptosis. The fibronectin-coated films, on the other hand, accommodated most of the healthy cells. This may arise due to the presence of fewer cells in a smaller area (as the fibronectin-coated films are reasonably hydrophobic, contact angle $68^{\circ}$; Figure 3 ), whereas the amount of nutrition (volume of complete media) was the same in every other case (controls and arginine-coated films). So, the amount of media was probably sufficient for the fewer cells present in the case of the fibronectin-modified films to thrive. On the other hand, in the case of surface modification by arginine, the amount of media was not adequate for providing nutrition for a larger number of cells that get adhered initially. We quantified the green (corresponding to live cells) and red (corresponding to dead cells) fluorescence intensities from several images. The normalized (with respect to area) plotting indicated an increase in the acridine orange fluorescence (green) on arginine- and fibronectin-coated polymers, indicating more number of live cells. At the same time, the higher dead cell population in bare polymers was proved by a higher red emission by them (Figure S7A).

To determine the extent of cellular proliferation, both cell counting and imaging experiments were performed. The cell counting at different intervals shows that the approximate number of cells adhered to both arginine- and fibronectincoated surfaces were very similar (Figure 8), keeping in mind the reported doubling time of L929 cells. ${ }^{22}$ According to both fluorescence microscopy (Figure 8) and live-dead assay (Figure 6), both for arginine- and fibronectin-coated films, the cellular density was higher. The quantification of green fluorescence (coming from the healthy cells containing fluorescein) and DAPI did show a similar trend as found in the microscopy experiment (Figure S7B). Thus, the results from several experiments clearly prove that the arginine- and fibronectin-coated films behave very similarly when it comes to viable cells accommodation. In the cellular proliferation study (Figure 5), using cell counting with Trypan blue, the arginineand fibronectin-coated films showed very comparable cell counts that were significantly superior to control surfaces (pristine PEI surface, gold nanoparticle-coated surface). Cell viability (by mitochondrial activity) using resazurin as an indicator also showed that at the end of 2 days, both arginine- and fibronectin-coated films showed a remarkably higher viable cell population in comparison to the control samples (Figure 5). Finally, the expression of F-actin (Figure 9; which indicates the healthy state of the cells) shows that the cells on fibronectin-coated surfaces were well stretched and prominent, which definitely arises due to the availability of the preferable RGD sequence. Interestingly, a careful observation (Figure S8) in the higher-magnification images revealed that in the argininecoated films as well, the F-actin is equally well expressed.

The reason behind the considerably excessive density of cells in arginine-coated films in comparison to untreated controls can be supported by the hydrophilicity generated by the arginine coating. But being as good as the fibronectin-coated film is really unanticipated. We speculate a 3-fold reason behind this phenomenon. The aginine-coated films are blessed with two special properties: (i) increased surface roughness (Figure S3), hence hydrophilicity (contact angle data from Figure 2), and (ii) surface charge. These are supportive of cellular spreading on the surface-coated films. ${ }^{23,24}$ Several literature reports stress upon the fact that surface polarization and roughness are crucial for cellular adhesion. ${ }^{25}$ Numerous reports compared the cellular entry of positively and negatively charged nanoparticles and found that the increasingly rough surface also encourages a "foothold" of a cell seeded on a material surface. ${ }^{26,27}$ Moreover, very fast spreading of water or media containing cells provides enough space in between cells for the healthy development in future and/or cellular migration. ${ }^{28}$ This effectively is a "low seeding density experiment", which in one way should help the proliferation of adhering cells like fibroblast. Instead, the fibronectin-coated films have the integrin-binding RGD sequence, which strongly encourages cells to bind to it, but at the same time, these films being hydrophobic, they do not allow the cells to spread over a larger surface area. As a result, during incubation, only a few cells adhere to the fibronectin-coated films because of space constraints. Thus, whereas fibronectin carries the RGD sequence and hence is friendly toward the cells, the argininecoated films have the material advantages in terms of surface roughness, low contact angle, and positive charge so much that we believe they behave very similarly to the positive effects of the RGD sequence and ultimately become almost equally effective when it comes to viable cell accommodation. The equivalent efficacy of both the films is also reflected in collagen production (Figure 10), where both arginine- and fibronectincoated films produce a comparable amount of collagen. Also worth mentioning is the fact that in the event of seeding, $2 \mathrm{~h}$ incubation and aspiration of cells on the films is considered to be a mimic of polymeric implant installation in the body, where new cells are suppose to adhere in a flowing system rather than adherence aided by gravity (a general in vitro practice).

\section{CONCLUSIONS}

Herein, we reported the modification of a surface hydrophobic nature of polyetherimide film to a hydrophilic one by simple procedure involving the layer-by-layer assembly approach. Traditionally expensive sticky proteins separated from animal tissue (fibronectin, vitronectin, laminin, etc.) are used for improved effects on cell adherence. Conversely, we demonstrated here that a small molecule like arginine is capable of serving the same purpose in terms of total viable cells. Medical translation of this simple concept could help lower down the costs related to polymeric implant installation and avoid 
complications arising out of a hydrophobic scaffold implantation

\section{MATERIALS AND METHODS}

Polyetherimide (PEI) sold under the trade name Ultem 1000 was obtained from General Electrical Co., Schenectady, NY. Chloroauric acid was obtained from the Seisco Research Laboratory, India. Arginine, fibronectin (tissue culture grade), carboxyfluorescein succinimidyl ester (CFSE), 4',6-diamidino2-phenylindole (DAPI), phosphate-buffered saline (PBS) powder, resazurin, and propidium iodide (PI) were purchased from Sigma-Aldrich. The murine originated fibroblast cell line L929 was procured from the cell line repository of NCCS Pune, India. Dulbecco's modified Eagle's medium (DMEM) and fetal bovine serum were purchased from Invitrogen. The collagen estimation kit (K218) was purchased from Biovision. Trypan blue was purchased from Himedia. The F-actin marker Alexafluor 488 phalloidin conjugate was bought from Thermo Fischer Scientific.

Preparation and Surface Modifications of PEI Films. Casting of PEI Films. PEI (available as solid polymer beads) was weighed and made into a solution of $0.0675 \mathrm{~g} / \mathrm{mL}$ in dry chloroform. It was carefully poured into $150 \mathrm{~mm}$ clean dry grease free flat glass Petri dish and covered with aluminum foil. The overnight setting at room temperature yielded a transparent light yellow film.

Gold Nanoparticles Synthesis. Gold nanoparticles were synthesized by the Turkevich method, ${ }^{18}$ where $0.1 \mathrm{mM}$ chloroauric acid was reduced by $10 \mathrm{mg} / \mathrm{mL}$ citric acid under boiling water conditions. The particles were characterized by UV-vis and TEM.

Plasma Treatment. Plasma treatment was carried out using $\mathrm{N}_{2}+\mathrm{H}_{2}$ plasma at $60 \mathrm{~W}$ power for $20 \mathrm{~min}$. The flow ratio was maintained at $3: 2 \mathrm{H}_{2}$ to $\mathrm{N}_{2}$. The instrument employed was the Emitech Plasma Asher K1050X.

Gold Nanoparticle Coating on PEI Films. Freshly plasmatreated PEI films were dipped in citrate-stabilized gold nanoparticle sol at room temperature and stirred overnight. After some time, the gold sol was found to lose its wine red color, following which it was replenished until a uniform gold layer formed on the films.

Arginine Coating on Gold-Coated PEI Films. The goldcoated films obtained from the above step were immersed overnight in a solution of arginine $(4 \mathrm{~g} / \mathrm{L})$ in Milli-Q water. After incubation, the films were washed with PBS and carried forward for characterization or applications.

Fibronectin-Coating on Gold Nanoparticle Coated PEI Films. The gold nanoparticle coated films were soaked in 40 $\mu \mathrm{g} / \mathrm{mL}$ of fibronectin in PBS overnight at $4{ }^{\circ} \mathrm{C}$. All the films were washed with PBS and air dried before characterization.

Characterization of Surface-Modified Films. UV-Vis Spectrophotometry. In this experiment, the PEI films were cut into $1 \mathrm{~cm} \times 4 \mathrm{~cm}$ rectangular strips. After plasma treatment followed by gold nanoparticle coating, the dry films were placed in UV cuvettes. CARY 300 Conc UV-vis spectrophotometer was used for the collection of the UV-vis spectrum.

Detection of Surface Roughness by AFM. The surfacemodified films (gold nanoparticle coated film, arginine- and fibronectin-coated films) and control (pristine films) were placed on glass slides and scanned using Nano Wizard Atomic Force Microscopy by contact method.

IR Spectroscopy. The surface-modified films were subjected to characterization using solid-state IR spectroscopy. In this method, PerkinElmer Spectrum 2 spectrophotometer was used in attenuated total reflectance (ATR) mode to collect data using 40 scans in the wavenumber range $500-4000 \mathrm{~cm}^{-1}$. For both these surface-modified films, background with only PEI was also taken.

Surface Hydrophilicity by Contact Angle Measurement. Surface hydrophilicity was measured by dropping $10 \mu \mathrm{L}$ water droplets on the modified polymer surface at room temperature and atmospheric pressure. The data were collected from a minimum of $\sim 5$ locations and the average contact angle values were determined. Kruss drop shape analyser version 1.41-02 was used for this purpose.

Water Absorption Study. For water absorption studies, the films were prepared after dipping them in arginine and fibronectin solutions (as per method described in the Material and Method section). They were air dried followed by high vacuum exposure for $2 \mathrm{~h}$. Each film was weighed using a microbalance. Each film was separately dipped in water, taken out, wicked off using a tissue paper, and weighed in the microbalance. The weight difference is the amount of water adsorbed on the film.

Mechanical Stability. Tensile testing was performed on a TA Instruments dynamic mechanical analyzer (DMA, RSAIII). Stretching experiments were performed using the rectangular tension geometry for the PEI sheets (control, arginine-coated, fibronectin-coated). The samples were cut into dog bone shapes for tensile testing. They were then clamped vertically in the rectangular geometry and stretched at a constant rate of $0.01 \mathrm{~mm} / \mathrm{s}$. Young's modulus was determined from these experiments.

Cell Culture Experiments with Modified Films. Common Protocol for Cell Culture Experiment. In all the tissue culture experiments, nontreated sterile 24-well plates were used. Films (modified as well as controls) were cut into 15 $\mathrm{mm}$ diameter circles and sterilized under UV inside tissue culture hood for $30 \mathrm{~min}$ on each side. They were aseptically put into an untreated a 24-well plate. Ten thousand murine fibroblast L929 cells were suspended in $50 \mu \mathrm{L}$ complete medium and seeded on each film. After $2 \mathrm{~h}$ incubation, the medium was aspirated and washed with $100 \mu \mathrm{L}$ of complete medium. Following this, all the films were allowed to incubate with $500 \mu \mathrm{L}$ complete DMEM medium under tissue culture conditions for the mentioned period of time. The results from each experiment were plotted as a mean including \pm SE for $n=$ 3.

Sample preparation for imaging: For imaging experiments, the films were washed twice with PBS, fixed with 4\% PFA for $15 \mathrm{~min}$, followed by washing with PBS. The cells were incubated in $0.1 \%$ Triton X-100 in PBS for 5 min followed by washing (PBS). They were further incubated in $5 \%$ bovine serum albumin (BSA) for $20 \mathrm{~min}$ to avoid nonspecific binding. Further, they were incubated with appropriate dyes. Images pertaining to a single experiment were taken using equal exposure time.

Evaluation of Cellular Adhesion. Cell Adhesion Assay by Cell Counting (Trypan Blue Assay). In this experiment, the arginine-coated PEI films were aseptically put into an untreated 24-well plate. Ten thousand healthy L929 fibroblast cells dispersed in $50 \mu \mathrm{L}$ complete DMEM medium were seeded on each film. The films were washed after aspirating the media. This step was performed to exclude gravitationally precipitated but nonadhered cells. After each predetermined time interval, $50 \mu \mathrm{L}$ media was taken out of the film, consolidated (with 
washing liquid), and mixed with Trypan blue. The number of cells present in the aliquot were counted with the help of an inverted microscope. In this way, the cellular density of "unattached" cells was determined. The data were compared with the same number of cells seeded into each well of a 24well untreated tissue culture plate and normalized with the cell number used for seeding.

Cell Viability and Cytotoxicity. Cell Viability Assay (by Resazurin). Cell seeding and culture were performed as per the common protocol mentioned. At $48 \mathrm{~h}$, the cells were incubated with $100 \mu \mathrm{M}$ resazurin (in complete DMEM) and allowed to incubate for $6 \mathrm{~h}$ under cell culture condition. The relative amount of viable cells was estimated by reading the emission at $590 \mathrm{~nm}$ (excitation 530-560 nm). The readings of cells grown on surface-modified films were normalized with the reading from cells grown on the adhering plate and plotted in percent.

Live-Dead Assay by Fluorescence Microscopy. Fifty thousand healthy L929 cells in $50 \mu \mathrm{L}$ complete DMEM media were seeded on each surface-modified films and controls. After $2 \mathrm{~h}$, the media were aspirated and replenished with 500 $\mu \mathrm{L}$ complete DMEM media. At 2 days time point, the cells were washed with PBS, incubated in $0.1 \%$ Triton X-100 for 5 min in the dark, and 5\% BSA in PBS. The films were washed twice with PBS. Subsequently, they were incubated with the live-dead assay stain of $10 \mu \mathrm{L} 7.5 \mathrm{mM}$ propidium iodide, $1 \mu \mathrm{L}$ $0.67 \mathrm{mM}$ acridine orange in $1 \mathrm{~mL}$ complete DMEM media (for each well) under cell culture conditions for $1 \mathrm{~h}$. The wells were washed with PBS and directly viewed under Axio Observer Z1 Carl Zeiss microscope using green and red channels. Emission of both the colors representing live and dead cells were quantified, normalized with respect to area, and plotted.

Effects on Cellular Proliferation. Cell Proliferation Study by Cell Counting. Cell seeding and incubation were done according to the common protocol discussed. After 1 and 2 days incubation under cell culture conditions, the media were aspirated. The wells were washed twice with PBS and the films were trypsinized with $100 \mu \mathrm{L}$ Trypsin ethylenediaminetetraacetic acid (for each film). After proper dilutions of the aliquots, the cell numbers were counted with the help of Trypan blue stain and inverted microscope. The plotted data were normalized with respect to the number of cells seeded.

Cell Populations on Differently Treated Surfaces by Fluorescence Microscopy. Cell seeding was performed according to the common protocol discussed. At $48 \mathrm{~h}$ time point, the cells were fixed and blocked. They were treated with CFSE in PBS $(5 \mu \mathrm{M})$ for $15 \mathrm{~min}$ at room temperature in the dark followed by washing with PBS. The films were incubated with $300 \mathrm{nM}$ DAPI at room temperature in the dark for 4 min followed by another brief PBS wash. They were mounted on clean glass slides with mounting media, sealed, and observed using Axio Observer Z1 Carl Zeiss microscope using green and cyan filters. The emission of fluorescein and DAPI from adhered cells were quantified, normalized with respect to area, and plotted.

Cell Morphology and Actin Cytoskeleton Staining. The cells were seeded according to the protocol discussed. They were incubated for $24 \mathrm{~h}$ in complete DMEM media. After two times, the PBS wash films were fixed and blocked. The actin filaments were stained by 100 times diluted Alexafluor 488 phalloidin (in PBS) incubation in dark for $30 \mathrm{~min}$ at room temperature. The cell nuclei were counterstained with DAPI at $300 \mathrm{nM}$ concentration for $4 \mathrm{~min}$ at room temperature (in dark) followed by two times wash in PBS. The films were directly placed on a coverslip and the images were captured using green and cyan filters by epifluorescence microscopy using an Axio Observer Z1 Carl Zeiss microscope.

Collagen Production Assay. The L929 cells, $1 \times 10^{6}$, were suspended in $200 \mu \mathrm{L}$ complete media and seeded on surface-modified UV-sterilized films (diameter $35 \mathrm{~mm}$ ) kept in 6-well plates. The media were aspirated after $2 \mathrm{~h}$ and $2 \mathrm{~mL}$ of fresh complete media was added to each well. At $24 \mathrm{~h}$, the media were changed. The aspirated media were saved. At 2 days time point, the media were aspirated and $1 \mathrm{~mL}$ spent media (from the combination of both days media) of each well was stored. After washing the films two times with sterile PBS, the cells were scraped off using a cell scraper and $400 \mu \mathrm{L}$ PBS. From the cells, collagen was estimated by lysing followed by oxidation. From the spent media, protein was precipitated, oxidized, and estimated according to the protocol mentioned in the collagen estimation kit $\mathrm{K} 218$ from Biovision. The amount of collagen generated in each sample was estimated by a calibration curve. The data plotted were normalized with the amount of collagen present on the bare film and presented in percent. From the data points (Figure 10), it may seem that the fibronectin-coated films have a higher amount of collagen formed. Genuinely, the difference (Figure 10A, third and fourth columns) is not significant as proved by a $t$-test using Excel.

\section{ASSOCIATED CONTENT}

\section{Supporting Information}

The Supporting Information is available free of charge on the ACS Publications website at DOI: 10.1021/acsomega.8b00215.

Characterization of gold nanoparticles by UV-vis spectrophotometry, TEM; characterization of arginineand fibronectin-coated PEI films by UV-vis, AFM, and IR; water absorption study, mechanical stability analysis, fluorescence intensity calculation, and actin cytoskeleton staining by fluorescence microscopy (PDF)

\section{AUTHOR INFORMATION}

\section{Corresponding Author}

*E-mail: pl.bhagavatula@ncl.res.in. Tel: 91-20-25902013. Fax: 91-2025902636.

ORCID

Bhagavatula L. V. Prasad: 0000-0002-3115-0736

Notes

The authors declare no competing financial interest.

\section{ACKNOWLEDGMENTS}

P.S. thanks DST-WOSA grant (grant number SR/WOS-A/CS94/2012) for fellowship and financial support. B.L.V.P. thanks CSIR, New Delhi, for the financial support through the M2D (CSC0134) project. The authors thank Ms Sneha of IISER Pune, Mr Shebeeb, and Ms S. Karthika from CSIR-NCL for operating AFM, contact angle instruments, and DMA analyzer, respectively.

\section{REFERENCES}

(1) Dhandayuthapani, B.; Yoshida, Y.; Maekawa, T.; Sakthi Kumar, D. Polymeric Scaffolds in Tissue Engineering Application: A Review. Int. J. Polym. Sci. 2011, No. 290602.

(2) Can, Z. Z.; Ercocen, A. R.; Apaydın, I.; Demirseren, E.; Sabuncuoglu, B. Tissue Engineering of high density porous polyethylene implant for three dimensional reconstruction: An experimental study. Scand. J. Plast. Reconstr. Surg. 2000, 34, 9-14. 
(3) James, S. P.; Oldinski, R. K.; Zhang, M.; Schwartz, H. UHMWPE Biomaterials Handbook; Elsevier, 2009; pp 259-276.

(4) Deshpande, S.; Munoli, A. Long-term results of high-density porous polyethylene implants in facial skeletal augmentation: An Indian perspective. Indian J. Plast. Surg. 2010, 43, 34-39.

(5) Kim, Y. H.; Jang, T. Y. Porous high-density polyethylene in functional rhinoplasty: Excellent long-term aesthetic results and safety. Plast. Surg. 2014, 22, 14-17.

(6) Fernandez-Bueno, I.; Di Lauro, S.; Alvarez, I.; Lopez, J. C.; Garcia-Gutierrez, M. T.; Fernandez, I.; Larra, E.; Pastor, J. C. Safety and Biocompatibility of a New High-Density Polyethylene-Based Spherical Integrated Porous Orbital Implant: An Experimental Study in Rabbits. J. Ophthalmol. 2015, No. 904096.

(7) Khalili, A. A.; Ahmad, M. R. A Review of Cell Adhesion Studies for Biomedical and Biological Applications. Int. J. Mol. Sci. 2015, 16, 18149-18184.

(8) Lotfi, M.; Nejib, M.; Naceur, M. Cell Adhesion to Biomaterials: Concept of Biocompatibility. Adv. Biomater. Sci. Biomed. Appl. 2013, $10.5772 / 53542$.

(9) Montaño-Machado, V.; Chevallier, P.; Mantovani, D.; Pauthe, E. On the potential for fibronectin/phosphorylcholine coatings on PTFE substrates to jointly modulate endothelial cell adhesion and hemocompatibility properties. Biomatter 2015, 5, No. e979679.

(10) Agarwal, R.; González-García, C.; Torstrick, B.; Guldberg, R. E.; Salmerón-Sánchez, M.; García, A. J. Simple coating with fibronectin fragment enhances stainless steel screw osseointegration in healthy and osteoporotic rats. Biomaterials 2015, 63, 137-45.

(11) He, L.; Tang, S.; Prabhakaran, M. P.; Liao, S.; Tian, L.; Zhang, Y.; Xue, W.; Ramakrishna, S. Surface modification of PLLA nanoscaffolds with laminin multilayer by LbL assembly for enhancing neurite outgrowth. Macromol. Biosci. 2013, 13, 1601-1609.

(12) Mehr, N. G.; Hoemann, C. D.; Favis, B. D. Chitosan surface modification of fully interconnected $3 \mathrm{D}$ porous poly ( $\varepsilon$-caprolactone) by the LbL approach. Polymer 2015, 64, 112-121.

(13) Shi, Q.; Qian, Z.; Liu, D.; Liu, H. Surface Modification of Dental Titanium Implant by Layer-by-Layer Electrostatic Self-Assembly. Front. Physiol. 2017, 8, No. 574.

(14) https://forschung-sachsen-anhalt.de/project/biomimeticsurface-modification-tissue-11863.

(15) D’Britto, V.; Tiwari, S.; Purohit, V.; Wadgaonkar, P. P.; Bhoraskar, S. V.; Bhonde, R. R.; Prasad, B. L. V. Composites of plasma treated poly(etherimide) films with gold nanoparticles and lysine through layer by layer assembly: a "friendly-rough" surface for cell adhesion and proliferation for tissue engineering applications. J. Mater. Chem. 2009, 19, 544-550.

(16) Sengupta, P.; Surwase, S. S.; Prasad, B. L. V. Modification of porous polyethylene scaffolds for cell attachment and proliferation. Int. J. Nanomed. 2018, 13, 87-90.

(17) Sengupta, P.; Prasad, B. L. V. Surface Modification of Polymeric Scaffolds for Tissue Engineering Applications. Regenerative Engineering and Translational Medicine 10.1007/s40883-018-0050-6.

(18) Turkevich, J.; Stevenson, P. C.; Hillier, J. A study of the nucleation and growth processes in the synthesis of colloidal gold. Discuss. Faraday Soc. 1951, 11, 55-75.

(19) Hong, J.; Prawer, S.; Murphy, A. B. Plasma Catalysis as an Alternative Route for Ammonia Production: Status, Mechanisms, and Prospects for Progress. ACS Sustainable Chem. Eng. 2018, 6, 15-31.

(20) Jiao, Y.; Xu, J.; Zhou, C. Effect of ammonia plasma treatment on the properties and cytocompatibility of a poly(L-lactic acid) film surface. J. Biomater. Sci. Polym. Ed. 2012, 23, 763-777.

(21) Kleinhans, C.; Barz, J.; Wurster, S.; Willig, M.; Oehr, C.; Müller, M.; Walles, H.; Hirth, T.; Kluger, P. J. Ammonia plasma treatment of polystyrene surfaces enhances proliferation of primary human mesenchymal stem cells and human endothelial cells. Biotechnol. J. 2013, 8, 327-337.

(22) Theerakittayakorn, K.; Bunprasert, T. Differentiation Capacity of Mouse L929 Fibroblastic Cell Line Compare With Human Dermal Fibroblast World Academy of Science, Engineering and Technology International Journal of Medical and Health Sciences 2011, 5251.
(23) Finke, B.; Luethen, F.; Schroeder, K.; Mueller, P. D.; Bergemann, C.; Frant, M.; Ohl, A.; Nebe, B. J. The effect of positively charged plasma polymerization on initial osteoblastic focal adhesion on titanium surfaces. Biomaterials 2007, 28, 4521-4534.

(24) Khang, D.; Kim, S. Y.; Liu-Snyder, P.; Palmore, G. T. R.; Durbin, S. M.; Webster, T. J. Enhanced fibronectin adsorption on carbon nanotube/poly(carbonate) urethane: Independent role of surface nano-roughness and associated surface energy. Biomaterials 2007, 28, 4756-4768.

(25) Miller, D. C.; Thapa, A.; Haberstroh, K. M.; Webster, T. J. Endothelial and vascular smooth muscle cell function on poly(lacticco-glycolic acid) with nano-structured surface features. Biomaterials 2004, 25, 53-61.

(26) Kim, D.-H.; Provenzano, P. P; Smith, C. L.; Levchenko, A. Matrix nanotopography as a regulator of cell function. J. Cell Biol. 2012, 197, 351-360.

(27) Ranella, A.; Barberoglou, M.; Bakogianni, S.; Fotakis, C.; Stratakis, E. Tuning cell adhesion by controlling the roughness and wettability of 3D micro/nano silicon structures. Acta Biomater. 2010, 6, 2711-2720.

(28) Selhuber-Unkel, C.; Erdmann, T.; Lopez-Garcia, M.; Kessler, H.; Schwarz, U. S.; Spatz, J. P. Cell adhesion strength is controlled by intermolecular spacing of adhesion receptors. Biophys. J. 2010, 98, $543-551$. 